\title{
Effect of omega-3 plus methylphenidate as an alternative therapy to reduce attention deficit- hyperactivity disorder in children
}

\author{
Soleiman Mohammadzadeh, MD', Narmin Baghi, MD², Fayegh Yousefi, PhD², Bahar Yousefzamani, PhD ${ }^{3}$ \\ ${ }^{1}$ Child and Adolescent Psychiatrist, Neurosciences Research Center, Kurdistan University of Medical Sciences, Sanandaj, Iran \\ ${ }^{2}$ Neurosciences Research Center, Kurdistan University of Medical Sciences, Sanandaj, Iran \\ ${ }^{3}$ Besat Hospital, Kurdistan University of Medical Sciences, Sanandaj, Iran
}

Background: Attention deficit-hyperactivity disorder (ADHD) is one of the most common chronic behavioral disorders in school-aged children.

Purpose: This study aimed to evaluate the effect of omega-3 supplementation as an alternative therapy for ADHD, which can be caused by vitamin and mineral deficiencies.

Methods: This was a double-blinded clinical trial study. Sixty-six children with ADHD (aged 6-12 years) referred to our child and adolescent psychiatric educational and therapeutic clinic were selected based on Diagnostic and Statistical Manual of Mental Disorders, Fourth Edition, Text Revision criteria. Instruments including the Parent ADHD Rating Scale were used to assess ADHD at 0, 2, 4, and 8 weeks during the study.

Results: The results showed no statistically significant difference between the methylphenidate with omega-3 group and methylphenidate with placebo group based on the Parents ADHD Rating Scale between week $0(P \geq 0.96)$ and week $8(P \geq 0.75)$. There were no significant intergroup differences between the Inattention $(P \geq 0.48)$ and hyperactivity/impulsivity $(P \geq 0.80)$ subscale scores on the Parents ADHD Rating Scale. The most common drug complications in the methylphenidate with placebo and methylphenidate with omega-3 groups were anorexia (27 [54\%] vs. 41 [60.29\%], respectively) and diarrhea (10 [20\%] vs. $8[11.76 \%]$, respectively), but the differences were not statistically significant ( $P>$ 0.05).

Conclusion: Our results demonstrate that a specific dose of omega-3 for 8 weeks had no effect on ADHD.

Key words: Attention deficit-hyperactivity disorder, Omega-3 supplement, Child

\section{Key message}

Question: Is the omega-3 supplementation with methylphenidate as an alternative therapy effect on the reduce $A D H D$ in children?

Finding: The results showed that there was no statistically significant difference between the methylphenidate with omega-3 group and methylphenidate with placebo $(P \geq 0.96)$.

Meaning: The results of our study showed that a specific dose of omega- 3 for 8 weeks had no effect on the treatment of $A D H D$

\section{Introduction}

Attention deficit-hyperactivity disorder (ADHD) is one of the most common neurodevelopment disorders in children, adolescents, and adults with attention deficits, hyperactivity, and impulsive behaviors ${ }^{1)}$. ADHD occurs in 5\% to $12 \%$ of children. According to reports, about
Corresponding author: Fayegh Yousefi, PhD Department of Psychiatry, Faculty of Medicine, Kurdistan University of Medical Science, Sanandaj, Iran

Tel: $+98-66-64640$

Fax: +98-66-64674

E-mail: f.yousefi@muk.ac.ir

https://orcid.org/0000-0001-9192-0926

Received: 1 September, 2018

Revised: 28 April, 2019

Accepted: 15 May, 2019
Copyright (C) 2019 by The Korean Pediatric Society

This is an open-access article distributed under the terms of the Creative Commons Attribution NonCommercial License (http://creativecommons.org/ licenses/by-nc/4.0/) which permits unrestricted noncommercial use, distribution, and reproduction in any medium, provided the original work is properly cited. 
59\% of clients referring to mental health services suffer from it. ${ }^{2)}$ It causes high costs to global health. ${ }^{3)}$

The main cause of the ADHD disorder is currently unknown, but in various studies, it has been linked to genetics, injury to the fetus, parturition complications, low birth weight ${ }^{4)}$ and smoking during pregnancy.

Over the past 2 decades, attention has been paid to the effects of diet on hyperactivity. Researchers have reported that various aspects of the child's diet including food additives, refined sugars, food allergies, and metabolism of minerals and fatty acids can have a negative impact on individual's behavior. ${ }^{6,7}$

This disorder causes many problems at home, in school, and in social settings. It may lead to academic problems, increased accidents, increased drug abuse, increased defiant and delinquent behaviors, and increased risk of depression and anxiety which necessitate treatment. ${ }^{8,9}$ For example, findings suggest that nearly 25\% of affected youth will not respond to above-mentioned medication (stimulants such as methylphenidate and other stimulants), or that they will discontinue usage due to negative side effects, most notably insomnia and loss of appetite. ${ }^{10-12)}$

Therefore, sometimes, instead of taking methylphenidate, other medications are considered.

Omega-3 supplement is one of the treatments used in ADHD. There is little information on its effectiveness. In a 2007 study, it has been reported that the use of Omega-3 supplements has significantly reduced ADHD symptoms in children. ${ }^{13)}$

Daily supplementation of omega-3 has led to low to moderate improvements in ADHD symptoms in several studies. ${ }^{14-16)}$ In a study conducted in 2009, its effectiveness has not been proven in children with $\mathrm{ADHD}{ }^{17)}$ In some studies, the reduced serum omega- 3 levels in children and adolescents with ADHD have been shown. ${ }^{7,18,19)}$ The main neurobiological mechanism of the omega-3 supplementation is quite clear. Omega-3 plays an important role in cell membrane elasticity and myelination resulting in better transmission of neural signals. ${ }^{20)}$ Studies have shown that cell membrane function and transfer of neurotransmitters are impaired due to the deficiency of the essential fatty acids of eicosapentaenoic acid (EPA) and docosahexaenoic acid. ${ }^{6,20)}$

In animal studies, it has been shown that dopamine and serotonin neurotransmitters have decreased in mice with low levels of omega-3. ${ }^{21,22)}$ Low levels of omega-3 in the frontal cortex of the mice are associated with hyperactivity and impulsivity. ${ }^{23)}$ In human studies, dopamine and cognition dysfunction have been proven in ADHD subjects. Given these cases, it is likely that omega-3 plays an important role in treating this disorder. ${ }^{24,25}$

Therefore, in a double-blinded clinical trial, the researchers investigated the efficacy of omega-3 supplementation as an adjunct therapy with methylphenidate in ADHD patients. Our hypothesis was that the combination of omega- 3 with methylphenidate is more effective than methylphenidate with placebo.

\section{Methods}

This study was a double-blinded clinical trial. The study population consisted of children with ADHD referred to the children and adolescent psychiatric clinic of Besat Hospital in Sanandaj, Iran, 2016. The study population consisted of 66 children ( 49 boys and 17 girls) aged 6 to 12 years old who were selected through simple random sampling. Also, the sample size based on $\alpha=0.05$ and $\beta=$ 0.20 was determined. The randomization list was made using a computerized random-number generator based on a randomnumber Table.

Of these, 6 were excluded. The drops occurred at the end of the second week. Two patients in the methylphenidate and omega-3 groups were unwilling to take medication. Four patients in the methylphenidate and placebo group left the study. One of them was due to seizure and 3 others due to gastrointestinal complications during drug use. Finally, 60 subjects' results were analyzed are shown in Fig. 1.

The ADHD was diagnosed by a psychiatrist of children and adolescents based on Diagnostic and Statistical Manual of Mental Disorders, Fourth Edition, Text Revision (DSM-IV-TR) criteria. A structured clinical interview with Kiddie Schedule for Affective Disorders and Schizophrenia was used to reject other disorders.

Patients were from all ADHD subtypes and new ones. The parents of children described the ADHD symptoms in accordance with the DSM-IV-TR criteria. Children who had a history of supplementation with omega-3 in at least the last 6 months, the presence of any known physical illness, mental disability, known psychiatric disorders (autism spectrum disorders, schizophrenia, intellectual impairment, and other psychiatric disorders), seizure, any psychiatrist comorbidity which needs treatment and children with suicide ideas were excluded.

Those who were willing to participate in the study entered it after a brief explanation of stages of study, receiving written consent from parents and oral consent of children. The agreement was designed in accordance with the ethical and legal regulations of Iran, in accordance with the Helsinki Statement (1975) and the 2008 edition of Human Resource Research. ${ }^{26)}$ In addition, this study was registered in IRCTID: IRCT2016060128182N2 (http://irct.ir/) on the Iranian clinical trial site. Also, it was approved by the Ethics

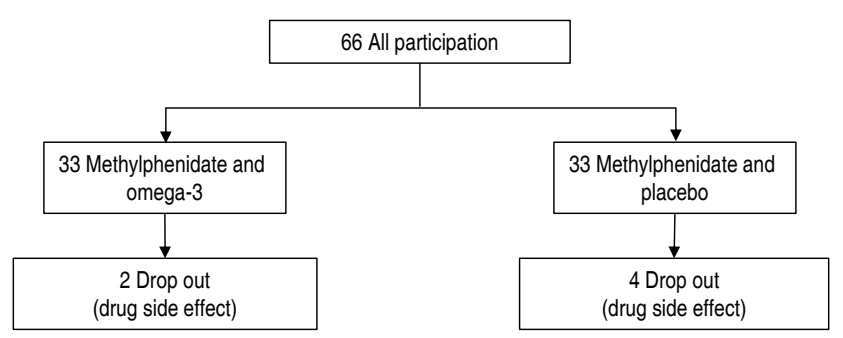

Fig. 1. Flow chart of the participant selection process. 
Committee of Kurdistan University of Medical Sciences (approval number: IR.MUK. REC.1394.265).

Patients began treatment after a structured clinical psychiatric interview and physical examination. Physical examination was performed for heart rate, blood pressure, and weight before the treatment. The demographic information checklists were filled after selecting the samples. The subjects were randomized divided into 2 groups and they referred with a code to the pharmacist. In addition, the patient, parents and the person who administrated the medications, were blinded, while the pharmacist was unblinded. Omega-3 EPA capsules (180 mg) and DHA (120 mg) and placebo (olive oil) were given to patients as pockets ( 1 and 2) as the same gel-shaped capsules produced by the Zahrawi Tabriz-Iran drug factory. One group of patients was treated with methylphenidate with an omega-3 supplement and one group was treated with Methylphenidate with placebo. Methylphenidate (10 mg) was prescribed as half a tablet at 8:00 AM and the other half at 4:00 PM daily in the first week and from the second week, 2 tablets (1 at 8:00 AM, 1 a 4:00 PM) based on the patient's tolerance. Given the need and tolerance of patients who were above $30 \mathrm{~kg}$, Methylphenidate was given up to $30 \mathrm{mg}$. One omega-3 capsule was given in the first week and one each morning in the placebo group. Then, from the second week, one omega-3 capsule was given in the morning and one was given in the afternoon and also it was given in placebo group similarity. For the participants, the ADHD Rating Scale (parents) test was completed before the start of the treatment and at weeks 2, 4, and 8 to determine the severity of ADHD symptoms.

\section{Parent's ADHD Rating Scale}

As a reliable and valid tool, it is used to assess the severity of $\mathrm{ADHD}$ symptoms and the rate of response to treatment in children and adolescents, which has 2 versions of parents and teachers. The purpose of the mentioned instrument was to deliver a method for obtaining teacher and parent ratings for clinical professionals, according to the frequency of each of the ADHD symptoms based on DSM-IV criteria.

This scale has 18 phrases in attention deficit and hyperactivity/ impulsivity. Eighteen items were written in such a way that, as briefly as possible, they represent DSM-IV criteria.

The respondent rate each item on a 4-point Likert-scale (0 to 3). Attention deficit items included even phrases and hyperactivity/ impulsivity included odd items. This has been done to reduce the bias in response. From the results of this scale, 3 scores (attention deficit, hyperactivity/impulsivity, and total) are obtained. The raw score of attention deficit is calculated by aggregating even scores, and the hyperactivity/impulsivity raw score is calculated by summing up the odd scores. The total raw score is calculated by adding the raw scores of its 2 subscales. Raw scores are converted to percentage points using the normative table scale based on the age and sex of the child. The internal consistency of this scale was $92 \%$ and the test-retest reliability was $85 \%$ with a 4-week interval. In the present study, Tehrani Dost and Zargirinejad translated form (2003) was used. $^{27)}$

\section{Demographics checklists}

It includes variables such as age, sex, weight, parent's education level, parent's job, and family income.

\section{Drug complication checklist}

A checklist was used to investigate the possible side effects of the drug in which the side effects reported for omega-3 and methylphenidate supplements were registered. Positive complications were recorded in the checklist in the 2nd, 4th, and 8th weeks after the start of the study, and if other complications were seen, they were also recorded.

\section{Data analysis}

After collecting information, the data were entered into IBM SPSS Statistics ver. 20.0 (IBM Co., Armonk, NY, USA) and the percentage, mean, and standard deviation were used for descriptive statistics. For analyzing the hypotheses of the research, $t$ test and repeated measure analysis of variance (ANOVA) were used.

\section{Results}

The 2 groups did not have a significant difference in terms of demographic characteristics (except weight) include sex, age, type of $\mathrm{ADHD}$, parent's job, parent's education, and family income are shown in Table 1.

The results also showed that the most common drug complication between both groups was anorexia and diarrhea. Also, diarrhea was more common in the methylphenidate and placebo group in the 8th week. Four patients in the methylphenidate and placebo groups were excluded from study 1 because of seizures and 3 because of gastrointestinal complications and restlessness during drug use. Also, based on Fisher exact test, there was no significant relationship between the 2 groups of methylphenidate and placebo with methylphenidate and omega-3 in drug complications $(P>0.05)$ are shown in Table 2.

The results of repeated measure ANOVA for ADHD-rating scale parent/total within-group effects showed that there was no significant difference between the treatment groups ( $F=0.39, P \geq 0.75$ ). However, the mean scores of subjects decreased during the course of treatment. In other words, in both groups, the $\mathrm{ADHD}$-rating scale parent/total was reduced are shown in Table 3.

Moreover, there was no significant difference between the 2 groups regarding the between-groups comparison ( $F=0.14, P \geq 0.70$ ). Comparing each single trend in 2 groups at a single time showed that there was no statistically significant difference between the 2 
Table 1. Participants' demographic characteristics by ADHD treatment type

\begin{tabular}{|c|c|c|c|}
\hline Variable & $\begin{array}{l}\text { Methylphenidate } \\
\text { and placebo }\end{array}$ & $\begin{array}{l}\text { Methylphenidate } \\
\text { and omega-3 }\end{array}$ & $P$ value \\
\hline Age (yr) & $8.20 \pm 1.72$ & $7.7 \pm 1.65$ & $>0.50$ \\
\hline Sex & & & $>0.39$ \\
\hline Girl & $7(21.2)$ & 10 (33.3) & \\
\hline Boy & $26(78.8)$ & $23(69.7)$ & \\
\hline Wight (kg) & $27.69 \pm 5.33$ & $23.77 \pm 5.68$ & $<0.006$ \\
\hline Father's job & & & $>0.19$ \\
\hline Unemployed & $0(0)$ & $2(6.1)$ & \\
\hline Employed & $33(100)$ & $31(93.9)$ & \\
\hline \multicolumn{4}{|l|}{ Mother's job } \\
\hline Unemployed & $30(90.9)$ & 27 (81.8) & $>0.28$ \\
\hline Employed & $3(9.1)$ & $6(18.2)$ & \\
\hline Education level & & & $>0.72$ \\
\hline \multicolumn{4}{|l|}{ Father } \\
\hline >Diploma & $28(84.8)$ & $29(87.9)$ & \\
\hline$\leq$ Bachelor & $5(15.2)$ & $4(12.1)$ & \\
\hline Mother & & & $>0.69$ \\
\hline$\geq$ Diploma & $29(87.9)$ & $30(90.9)$ & \\
\hline$\leq$ Bachelor & $4(12.1)$ & $3(9.1)$ & \\
\hline Family income & & & $>0.80$ \\
\hline$\leq \$ 100$ & $16(48.5)$ & $17(51.5)$ & \\
\hline$>\$ 100$ & 17 (51.5) & $16(48.5)$ & \\
\hline Baseline Parent ADHD Rating Scale & $42.66 \pm 6.76$ & $42.75 \pm 6.79$ & $>0.96$ \\
\hline \multicolumn{4}{|l|}{ Drug dose (mg/day) } \\
\hline 1-Week dose & 10 & 10 & \\
\hline 2-Week dose & 20 & 20 & \\
\hline 4-, 6-, and 8-week doses & 20 & 20 & \\
\hline
\end{tabular}

Values are presented as mean \pm standard deviation or number (\%) unless otherwise indicated

ADHD, attention deficit-hyperactivity disorder.

Boldface indicates a statistically significant difference with $P<0.05$.

groups are shown in Table 3.

The results of repeated measure ANOVA for within-group effects of attention-deficit subscale showed that there was no significant difference during the weeks of treatment $(F=0.79, P \geq 0.48)$. However, the mean scores of subjects have decreased over the course of the treatment indicating that the mean of attention-deficit was reduced in both groups are shown in Table 3.

Also, there was no significant difference between the 2 groups regarding the between-groups comparison $(F=0.32, P \geq 0.86)$. Comparing each single trend in 2 groups at a single time showed that there was no statistically significant difference between the 2 groups are shown in Table 3.

The results of repeated measure ANOVA for within-group effects of hyperactivity/impulsivity subscale showed that there was no significant difference between 2 groups during weeks $(F=0.27, P \geq$
Table 2. Comparison of complications observed in the 2 groups during the study

\begin{tabular}{lccc}
\hline & $\begin{array}{c}\text { Methylphenidate } \\
\text { and placebo }\end{array}$ & $\begin{array}{c}\text { Methylphenidate } \\
\text { and omega-3 }\end{array}$ & $P$ value \\
\hline Type of complication & $1(2)$ & $2(2.9)$ & $>0.05$ \\
Nausea & $1(2)$ & $4(5.9)$ & \\
Vomiting & $10(20)$ & $8(11.8)$ & \\
Diarrhea & $1(2)$ & $1(1.5)$ & \\
Stomachache & $1(2)$ & $1(1.5)$ & \\
Dry mouth & $1(2)$ & $2(2.9)$ & \\
Drowsiness & $2(4)$ & $2(2.9)$ & \\
Insomnia & $1(2)$ & $1(1.5)$ & \\
Anxiety & $27(54)$ & $41(60.3)$ \\
Anorexia & $2(4)$ & $3(4.4)$ \\
Restlessness & $2(4)$ & $3(4.4)$ & \\
Irritability & $1(2)$ & $0(0)$ & \\
Seizure & &
\end{tabular}

Values are presented as number (\%).

Fisher exact test.

Table 3. Intergroup comparison of the total and subscale parent ADHD Rating Scale scores by week

\begin{tabular}{lcccc}
\hline Parent ADHD Rating Scale & $\begin{array}{c}\text { Methylphenidate } \\
\text { and placebo }\end{array}$ & $\begin{array}{c}\text { Methylphenidate } \\
\text { and omega-3 }\end{array}$ & $F$ & $P$ value \\
\hline Total & & & 0.39 & $\geq 0.75$ \\
$\quad$ Week (baseline) & $42.61 \pm 6.86$ & $42.51 \pm 7.18$ & & \\
2-Weeks & $34.90 \pm 8.50$ & $35.82 \pm 10.10$ & & \\
4-Weeks & $25.58 \pm 10.06$ & $25.75 \pm 10.78$ & & \\
8-Weeks & $15.77 \pm 8.21$ & $17.75 \pm 9.620$ & & \\
Inattention & & & 0.79 & $\geq 0.48$ \\
Week (baseline) & $22.25 \pm 2.82$ & $21.86 \pm 3.98$ & & \\
2-Weeks & $18.64 \pm 4.88$ & $18.48 \pm 4.438$ & & \\
4-Weeks & $14.06 \pm 5.37$ & $13.13 \pm 5.53$ & & \\
8-Weeks & $9.00 \pm 4.74$ & $9.79 \pm 4.81$ & & \\
Hyperactivity/impulsivity & & & 0.27 & $\geq 0.80$ \\
Week (baseline) & $20.35 \pm 5.59$ & $20.65 \pm 4.42$ & & \\
2-Weeks & $16.25 \pm 5.15$ & $17.34 \pm 5.75$ & & \\
4-Weeks & $11.51 \pm 5.56$ & $12.62 \pm 5.46$ & & \\
8-Weeks & $6.77 \pm 4.152$ & $7.96 \pm 4.92$ & & \\
\hline
\end{tabular}

Repeated-measures analysis of variance.

Values are presented as mean \pm standard deviation.

$\mathrm{ADHD}$, attention deficit-hyperactivity disorder.

0.80). However, the mean scores of subjects have decreased over the course of treatment, indicating that in both groups the mean of hyperactivity has decreased are shown in Table 3.

In addition, there was no statistically significant difference between the 2 groups regarding between-groups comparisons ( $F=0.65$, $P \geq 0.42$ ). In addition, comparing single trends in 2 groups at a single time showed that there was no statistically significant difference between the 2 groups are shown in Table 3 . 


\section{Discussion}

$\mathrm{ADHD}$ disorder is one of the most common psychiatric diseases occurring in 5\% to $12 \%$ of children.

The main cause of ADHD is unknown and many factors contribute to its development. One of the possible causes is the role of nutritional factors in the hyperactivity of children. The short age of fatty acids, i.e., omega 3 and 6, contribute to the development of $\mathrm{ADHD}$. One of the important roles of essential fatty acids is the communication between the brain cells.

Nerve cell membranes include phospholipids in which fatty acids are one of its main components. It is suggested that essential fatty acids affect the function of cell membranes and transmissions of neurotransmitters. Thus, these functions are disrupted by the lack of essential fatty acids. $^{7,28)}$

Regarding the role of norepinephrine and dopamine in the physiopathology of $\mathrm{ADHD}$, nerve stimulant drugs such as methylphenidate are known as a selective therapy. However, around 30\% of children do not respond to methylphenidate and there is a potential risk of abusing methylphenidate as it may be necessary to take at least one dose at school. ${ }^{\text {9) }}$ So, sometimes instead of taking methylphenidate, other medications include venlafaxine, ${ }^{29)}$ memantine ${ }^{30,31)}$ and bupropion ${ }^{32)}$ are prescribed.

A double-blinded clinical trial was conducted on ADHD children. The present study showed that omega-3 supplementation has no effect on the treatment of children with $\mathrm{ADHD}$, so, the study results were negative.

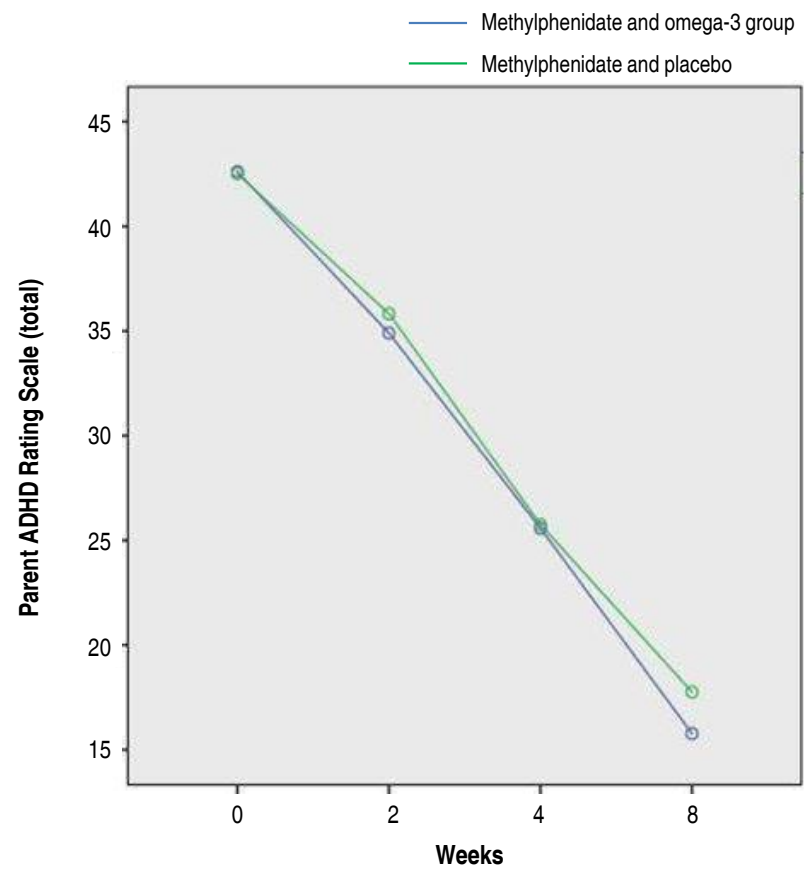

Fig. 2. Figure showing no significant intergroup differences during the study period $(\mathrm{f}=0.39, P \geq 0.75)$
Previous studies have shown that omega-3s are well tolerated by children and have minor side effects such as digestive discomfort and nose bleeding. ${ }^{33)}$ In a study by the Mexican Mental Health Center in children with $\mathrm{ADHD}$, children who received methylphenidate supplemented with omega-3 and -6 had lower needs for medication and lower side effects. ${ }^{34)}$ A review study of 25 clinical trials has shown that omega-3s are effective in 2 groups of patients. Firstly, people with mild ADHD. Secondly, people with severe ADHD for whom omega-3 supplement reduced the amount of stimulant drug and, in particular, the side effects of drugs. ${ }^{35)}$

In our study, the drug complications were almost similar in the methylphenidate with omega-3 groups and methylphenidate with placebo groups. However, some complications such as anorexia, diarrhea, and irritability were the most common complications between the 2 groups. Although, the rate of anorexia and diarrhea was higher in the control group. Also, diarrhea was more common in the methylphenidate and placebo groups in the 8th week. One case, the seizure was observed in the methylphenidate and placebo group, so that subject was excluded. Most patients easily tolerated complications and continued the study.

The 2 groups were similar in terms of clinical symptoms at the beginning of the study is shown in Fig. 2.

The results showed that there was no significant difference between the 2 groups of methylphenidate with omega- 3 and methylphenidate with placebo based on the parents ADHD Rating Scale for 8 weeks $(P=0.692)$. Also, there was no significant difference between attention-deficit $(P=0.86)$ and hyperactivity $(P=0.409)$ subscales in the parents $\mathrm{ADHD}$ rating scale. In other words, there was no difference between the effect of methylphenidate with omega-3 and methylphenidate with placebo on $\mathrm{ADHD}$ and between attention deficit and hyperactivity subscales.

In a 2009 study, 75 children and adolescents aged 8-18 years were randomly treated with omega-3 and -6 supplements for 3 months. They have not received any medication for 3 months. Most of these subjects did not respond to treatment and only $26 \%$ of people reported a reduction of more than $25 \%$ in ADHD symptoms. In the first 3 months, changes in all parameters were higher in the case group than the control group ( $P=0.04)$, and $47 \%$ responded to treatment after 6 months. ${ }^{17)}$

The results of our study are consistent with the result of the above study. In our study, there was no significant difference between the ADHD rating scales of attention deficit and hyperactivity in the 2 treatment groups during the 8 weeks.

In a review study (2011), the researchers evaluated the effectiveness of omega-3 fatty acid supplementation in children with ADHD. Omega-3 supplementation has had a dramatic effect on the recovery of ADHD symptoms in a few cases. The dosage of EPA in the supplement has been significantly associated with the degree of supplement efficacy. Overall, omega-3 supplementation had a modest effect compared to other available drugs for the treatment of $\mathrm{ADHD}$, such 
as psycho-stimulants or alpha-2 agonists in high doses. However, considering the harmful effects of omega-3 and its moderate effect on $\mathrm{ADHD}$, it seems logical to use as a reinforce of common drug or for patients (families) who do not accept other psychiatric interventions. $^{15)}$

The results of this study are not consistent with our study in terms of the effect of omega-3 on ADHD. The sample size of this study is very different from our study which may be one of the reasons for the difference in outcome. Alternatively, it can be the results of the difference in dosage of supplementation with omega-3, which was lower in our study. However, the results are consistent on the complications of omega-3 drugs compared to other drugs.

In a study, the effects of omega- 3 and -6 supplements have been evaluated in the treatment of methylphenidate-resistant ADHD children. This study was conducted on children aged 6 to 12 with ADHD who have been treated more than 6 months with methylphenidate and behavioral therapy.

According to their parents, there was no improvement in their behavior, educational status and learning. Children were randomly divided into 2 groups. One group received omega- 3 and -6 supplements and the other group received placebo. At 3 and 6 months after the start of the study, the treatment outcomes were assessed through the checklist given to the parents of children. There were statistically significant improvements in the case group in terms of: restlessness, aggression, fulfilling works and academic performance $(P<0.01){ }^{14)}$

There was no significant improvement between the 2 groups during the first 3 months of treatment, but after 6 months, significant improvement was observed in the case group in terms of attention, impulsive behavior and collaboration with family and teachers $(P<0.05){ }^{16)}$

In our study, there was no significant difference between ADHD Parent rating Scale subscales in the 2 treatment groups over a period of 8 weeks. However, the mean scores of attention deficit and hyperactivity of ADHD Rating Scales subscales decreased over the course of 8 weeks.

The reason for the difference in our results with previous studies may be the age of our subjects, which was lower, and the duration of the treatment, which was 2 months or because of the instrument.

In conclusion, although supplements and alternative therapies are widely used in the treatment of ADHD, the results of our study showed that omega-3 had no effect on ADHD with the specified dose and over a period of 8 weeks. Overall, the results of our study were negative. Based on that, treatment with omega-3 supplementation requires studies with higher doses and longer duration, and in this study omega-3 supplementation has not a dangerous complication and is easily tolerated. The limitation of this study includes, first, small sample size and imbalance of sex, second, no measurement of blood levels of EPA and docosahexaenoic acid because of to be not kets in experimental centers in our university in before and after treatment by omega 3. Third, the duration of the study was 8 weeks, which was a short time. Studies with longer duration are required. However, if the mentioned limitation were not, may be the results of this study will be different.

\section{Conflicts of interest}

No potential conflict of interest relevant to this article was reported.

\section{Acknowledgments}

The authors are thankful for the deputy and director of research and other collaborators who were involved in all stages of the research. In addition, we are thankful for all the patients who participated in this project. Also, this article has been extracted from the dissertation of Medical student titled:" The effect of omega-3 supplementation with methylphenidate as an alternative therapy to reduce the ADHD in children at Kurdistan University of Medical Sciences.

\section{References}

1. Antai-Otong D, Zimmerman ML. Treatment approaches to attention deficit hyperactivity disorder. Nurs Clin North Am 2016;51:199-211.

2. Connor DF. Preschool attention deficit hyperactivity disorder: a review of prevalence, diagnosis, neurobiology, and stimulant treatment. J Dev Behav Pediatr 2002;23(1 uppl):S1-9.

3. Rowland AS, Lesesne CA, Abramowitz AJ. The epidemiology of attention-deficit/hyperactivity disorder (ADHD): a public health view. Ment Retard Dev Disabil Res Rev 2002;8:162-70.

4. Mick E, Biederman J, Prince J, Fischer MJ, Faraone SV. Impact of low birth weight on attention-deficit hyperactivity disorder. J Dev Behav Pediatr 2002;23:16-22.

5. Mick E, Biederman J, Faraone SV, Sayer J, Kleinman S. Case-control study of attention-deficit hyperactivity disorder and maternal smoking, alcohol use, and drug use during pregnancy. J Am Acad Child Adolesc Psychiatry 2002;41:378-85.

6. Kidd PM. Attention deficit/hyperactivity disorder (ADHD) in children: rationale for its integrative management. Altern Med Rev 2000;5: 402-28.

7. Chen JR, Hsu SF, Hsu CD, Hwang LH, Yang SC. Dietary patterns and blood fatty acid composition in children with attention-deficit hyperactivity disorder in Taiwan. J Nutr Biochem 2004;15:467-72.

8. Rather YH, Sheikh AA, Sufi AR, Qureshi AA, Wani ZA, Shaukat TS. ADHD presenting as recurrent epistaxis: a case report. Child Adolesc Psychiatry Ment Health 2011;5:13.

9. Banaschewski T, Roessner V, Dittmann RW, Santosh PJ, Rothenberger A. Non-stimulant medications in the treatment of ADHD. Eur Child Adolesc Psychiatry 2004;13 Suppl 1:I102-16.

10. Barkley RA, McMurray MB, Edelbrock CS, Robbins K. Side effects of methylphenidate in children with attention deficit hyperactivity disorder: a systemic, placebo-controlled evaluation. Pediatrics 1990; 86:184-92. 
11. DuPaul GJ, RA Barkley, DF Connor. Stimulants. In: Barkley RA, edior. Attention deficit hyperactivity disorder: A handbook for diagnosis and treatment. 2nd ed. New York: Guilford Press; 1998. p. 510-51.

12. Elia J, Borcherding BG, Rapoport JL, Keysor CS. Methylphenidate and dextroamphetamine treatments of hyperactivity: are there true nonresponders? Psychiatry Res 1991;36:141-55.

13. Sinn N, Bryan J. Effect of supplementation with polyunsaturated fatty acids and micronutrients on learning and behavior problems associated with child ADHD. J Dev Behav Pediatr 2007;28:82-91.

14. Aben A, Danckaerts M. Omega-3 and omega- 6 fatty acids in the treatment of children and adolescents with ADHD. Tijdschr Psychiatr 2010;52:89-97.

15. Bloch MH, Qawasmi A. Omega-3 fatty acid supplementation for the treatment of children with attention-deficit/hyperactivity disorder symptomatology: systematic review and meta-analysis. J Am Acad Child Adolesc Psychiatry 2011;50:991-1000.

16. Perera H, Jeewandara KC, Seneviratne S, Guruge C. Combined $\omega 3$ and $\omega 6$ supplementation in children with attention-deficit hyperactivity disorder (ADHD) refractory to methylphenidate treatment: a doubleblind, placebo-controlled study. J Child Neurol 2012;27:747-53.

17. Johnson M, Ostlund S, Fransson G, Kadesjö B, Gillberg C. Omega-3/ omega- 6 fatty acids for attention deficit hyperactivity disorder: a randomized placebo-controlled trial in children and adolescents. J Atten Disord 2009;12:394-401.

18. Antalis CJ, Stevens LJ, Campbell M, Pazdro R, Ericson K, Burgess JR. Omega-3 fatty acid status in attention-deficit/hyperactivity disorder. Prostaglandins Leukot Essent Fatty Acids 2006;75:299-308.

19. Colter AL, Cutler C, Meckling KA. Fatty acid status and behavioural symptoms of attention deficit hyperactivity disorder in adolescents: a case-control study. Nutr J 2008 Feb 14;7:8. doi: 10.1186/1475-28917-8.

20. Bazinet RP, Layé S. Polyunsaturated fatty acids and their metabolites in brain function and disease. Nat Rev Neurosci 2014;15:771-85.

21. Chalon S. Omega-3 fatty acids and monoamine neurotransmission. Prostaglandins Leukot Essent Fatty Acids 2006;75:259-69.

22. Kodas E, Galineau L, Bodard S, Vancassel S, Guilloteau D, Besnard JC, et al. Serotoninergic neurotransmission is affected by n-3 polyunsaturated fatty acids in the rat. J Neurochem 2004;89:695-702.

23. Vancassel S, Blondeau C, Lallemand S, Cador M, Linard A, Lavialle M, et al. Hyperactivity in the rat is associated with spontaneous low level of $n-3$ polyunsaturated fatty acids in the frontal cortex. Behav Brain Res 2007;180:119-26.
24. Kirley A, Hawi Z, Daly G, McCarron M, Mullins C, Millar N, et al. Dopaminergic system genes in ADHD: toward a biological hypothesis. Neuropsychopharmacology 2002;27:607-19.

25. Durston S, Konrad K. Integrating genetic, psychopharmacological and neuroimaging studies: a converging methods approach to understanding the neurobiology of ADHD. Dev Rev 2007;27:374-95.

26. Carlson RV, van Ginneken NH, Pettigrew LM, Davies A, Boyd KM, Webb DJ. The three official language versions of the Declaration of Helsinki: what's lost in translation? J Med Ethics 2007;33:545-8.

27. Zhang S, Faries DE, Vowles M, Michelson D. ADHD Rating Scale IV: psychometric properties from a multinational study as a clinicianadministered instrument. Int J Methods Psychiatr Res 2005;14:186201.

28. Hawkey E, Nigg JT. Omega-3 fatty acid and ADHD: blood level analysis and meta-analytic extension of supplementation trials. Clin Psychol Rev 2014;34:496-505.

29. Amiri S, Farhang S, Ghoreishizadeh MA, Malek A, Mohammadzadeh S. Double-blind controlled trial of venlafaxine for treatment of adults with attention deficit/hyperactivity disorder. Hum Psychopharmacol 2012;27:76-81.

30. Mohammadi MR, Mohammadzadeh S, Akhondzadeh S. Memantine versus methylphenidate in children and adolescents with attention deficit hyperactivity disorder: a double-blind, randomized clinical trial. Iran J Psychiatry 2015;10:106-14.

31. Mohammadzadeh S, Ahangari TK, Yousefi F. The effect of memantine in adult patients with attention deficit hyperactivity disorder. Hum Psychopharmacol 2019;34:e2687.

32. Kuperman S, Perry PJ, Gaffney GR, Lund BC, Bever-Stille KA, Arndt S, et al. Bupropion SR vs. methylphenidate vs. placebo for attention deficit hyperactivity disorder in adults. Ann Clin Psychiatry 2001;13:129-34.

33. Königs A, Kiliaan AJ. Critical appraisal of omega-3 fatty acids in attention-deficit/hyperactivity disorder treatment. Neuropsychiatr Dis Treat 2016;12:1869-82.

34. Barragán E, Breuer D, Döpfner M. Efficacy and safety of omega-3/6 fatty acids, methylphenidate, and a combined treatment in children with ADHD. J Atten Disord 2017;21:433-41.

35. Bos DJ, Oranje B, Veerhoek ES, Van Diepen RM, Weusten JM, Demmelmair $\mathrm{H}$, et al. Reduced symptoms of inattention after dietary omega-3 fatty acid supplementation in boys with and without attention deficit/hyperactivity disorder. Neuropsychopharmacology 2015; 40:2298-306. 